\title{
Analysis of solar radius determination obtained by the modern CCD astrolabe of the Calern Observatory
}

\author{
A new approach of the solar limb definition \\ F. Chollet and V. Sinceac \\ Observatoire de Paris (DANOF/UMR 8630), 61 avenue de l'Observatoire, 75014 Paris, France
}

Received January 14; accepted June 16, 1999

\begin{abstract}
A semi-empirical model of solar images is presented here and used to derive the solar radius from astrolabe observations made at the Côte d'Azur Observatory (OCA), at the Calern station. This model was elaborated in order to remove a systematic effect existing in the measurements. This effect is caused by the center to limb darkening existing in the apparent solar light intensity and is magnified by atmospheric and instrumental effects. The result is that the apparent or observed solar radius is always smaller than the true one.
\end{abstract}

After a description of the observational methods used, a definition of the true solar radius is given and the model is described (Eqs. (1) or (3)). A new set of results is obtained using the model, which corresponds to observations made with a rotating shutter (used to separate the two solar images present in the focal plane). All the results are given for the unit distance.

This paper presents two series of solar apparent radius (see Table 1).

The first one (column derivative on Table 1), obtained in a previous analysis uses only numerical methods, is affected by the perturbing effects of the center to limb darkening, atmospheric turbulence and attenuation and conducts to the mean value $9599^{\prime \prime} 44 \pm 0$ "' 02 .

Taking account of all the informations given by the CCD observations, correlations between the Fried parameter $r_{0}$ and the derivative width, and between $r_{0}$ and the solar radius $R$, are found, in the results obtained by purely numerical methods. These results give us the possibility to evaluate, for $r_{0} \rightarrow \infty$, the corresponding values of the derivative width and the solar radius outside the terrestrial atmosphere. In these conditions, the mean solar radius is found to be equal to $9599^{\prime \prime} 63 \pm 0$ ". 08 .

The second series (column model on Table 1 ) obtained by the use of the presented model, conducts directly to a corrected mean result $R=959$.' $64 \pm 0$ ". 02 .

Send offprint requests to: F. Chollet
One can see that the corrected result of the first method agrees very well with the one obtained using the model, which has a better precision.

By the same way, the parameters $b$, which represent the slope of the solar limb near the true inflection point, and $p$, which define the slope of the darkening effect near the limb are given through and outside the atmosphere. As we hoped, the slope $b$ of the derivative is much greater outside than inside the atmosphere (about two times). The contrary occurs for $p$, as the darkening is increased by the presence of the atmosphere and dust ...

The comparison with other results and method shows that the model has the strong advantage to give directly the correct result without any supplementary correction. Some observations done without the rotating shutter at Calern, Rio de Janeiro and San Fernando Observatories will be analyzed in a near future using our model, applied to these existing data. We hope so to analyze the future observations from Antalya where a CCD astrolabe will start a new campaign.

Key words: Sun: general — Sun: oscillations - Sun: photosphere - Sun: radius — astrometry — atmospheric effects

\section{Introduction}

Since the years 70, astrometric observations of the Sun are done with the solar astrolabe at Calern station - Côte d'Azur Observatory (Laclare et al. 1980; Chollet 1981) to estimate its radius and the eventual variations of this parameter. The quality of the visual observations which give, since 1978, the best evaluation of the solar radius, urged us to increase the quality of the instrument and to begin studies in order to automate the observations. This is partially done now: the 
modern astrolabes use zerodur prisms and the observations are made using CCD video camera (Sinceac 1998; Sinceac et al. 1998a).

These observations have a great importance in many scientific domains. Firstly, the solar radius is an important parameter for the physical study of this star and its variations, if they are real, are not only important for the solar physics but also for celestial mechanics and the planets motion in relativistic gravitation theory.

In other way, if the quality of these observations can be extended to other objects as stars, planets and small planets, a connection between the existing spatial reference frames could be possible. Few experiments now in progress shows that these objectives are attainable (Kurzynska 1997; Popescu 1998). A first test done by the authors, with intensified video CCD camera, shows that objects of magnitude as high as 9 or 10 may be observable now.

But, it is not sufficient to have the best instrument, if the subsequent analysis of the observations is not carefully done. The aim of this work is to remove disruptive effects on the observations, which can be resumed as:

The apparent or observed solar radius is always smaller than the true one.

It is well known that a center to limb effect exists on solar images, which shows a decreasing of the solar light intensity (Allen 1973; Pecker \& Schatzman 1959). In the evaluation of the observed radius, this effect is amplified and modified by atmospheric turbulence and transmission.

The true radius needs to be defined. This necessary definition will be derived from one of the adopted parameters of the solar images model. Indeed, the amount of data obtained by CCD observations allows us to construct a model which depends only on 5 parameters. More, we will see that the adopted model gives a proof of the reality of the disruptive effects mentioned above. Taking account of the different effects acting to construct the final image on the CCD, we will define the solar radius as:

the semi-diameter of a large circular source of light emitting with constant intensity,

that is without center to limb effect. We precise here that the observed diameter is always a vertical one and that the observations are made at constant zenith distances.

In order to be clear, a description of observations methodology should be presented briefly (for details, see Sinceac 1998; Sinceac et al. 1998a). This description will help to understand the way followed to solve the problem.

\section{The observations methodology}

The astrolabe was elaborated in order to give two images of the same star which follow two symmetrical trajectories relatively to an horizontal line. The measurement principle is to find the instant when the two images of the star define a horizontal line. At this instant, the zenith distance of the star is exactly the one defined by a prism, equivalent to a double mirror, associated with a mercury surface. Finally, to find the transit time, the observational principle is to reconstruct the apparent zenith distance variations of each image as functions of time.

In the case of solar observations, we should find the reference points of two images of the same part of the solar limb. These points are the ones for which the tangential lines to the curves defined by the two images of the solar limb are horizontal. If we suppose that these points are known, the observation follows the same way as for the stars.

This principle is valid as well as for visual and for CCD observations. As the astrolabe shows two solar images during the transit, these images are superposed during half of the transit duration. In order to remove this effect, the observations were done, firstly, using a rotating shutter to acquire, alternatively, the direct image and the reflected one on the mercury.

Each image gives the distribution of the apparent solar intensity $I(x, y)$ relatively to the CCD frame. This frame is defined by the CCD lines (parallel to the $x$-axis) and columns (parallel to the $y$-axis). The CCD lines are vertical, due to the choice of the best resolution. The subsequent analysis is done in order to obtain the successive positions of the extremity of the vertical solar diameter (VSR) in the same CCD reference frame.

The sets of these positions, one for each successive direct or reflected image obtained during the limb transit, show the trajectories of the extremity of the VSR seen directly or reflected on mercury. These coordinates are functions of time $x_{\mathrm{d}}(t)$ and $y_{\mathrm{d}}(t)$ (direct images), and $x_{\mathrm{r}}(t)$ and $y_{\mathrm{r}}(t)$ (reflected images) relatively to the CCD frame. The knowledge of these functions gives us the possibility to determine:

- the transit time of the observed VSR;

- the correction on the CCD line inclination, and consequently the corresponding correction on the observed transit time;

- the pixel sizes on the sky (in arcseconds);

- the true extremity of the VSR along the limb.

The transit time is obtained simply when the two coordinates $y_{\mathrm{d}}(t)$ of the direct image and $y_{\mathrm{r}}(t)$ of the reflected one are equal. The coordinates $x_{\mathrm{d}}(t)$ and $x_{\mathrm{r}}(t)$ are not well determined but are useful to calculate, with a sufficient precision, the previous corrections.

Finally the comparison of the transit times obtained for each edge of the Sun, allows us to obtain the observed correction to the apparent solar diameter. Simple subsequent calculation gives the corrected apparent radius for the unit distance (1 AU). 


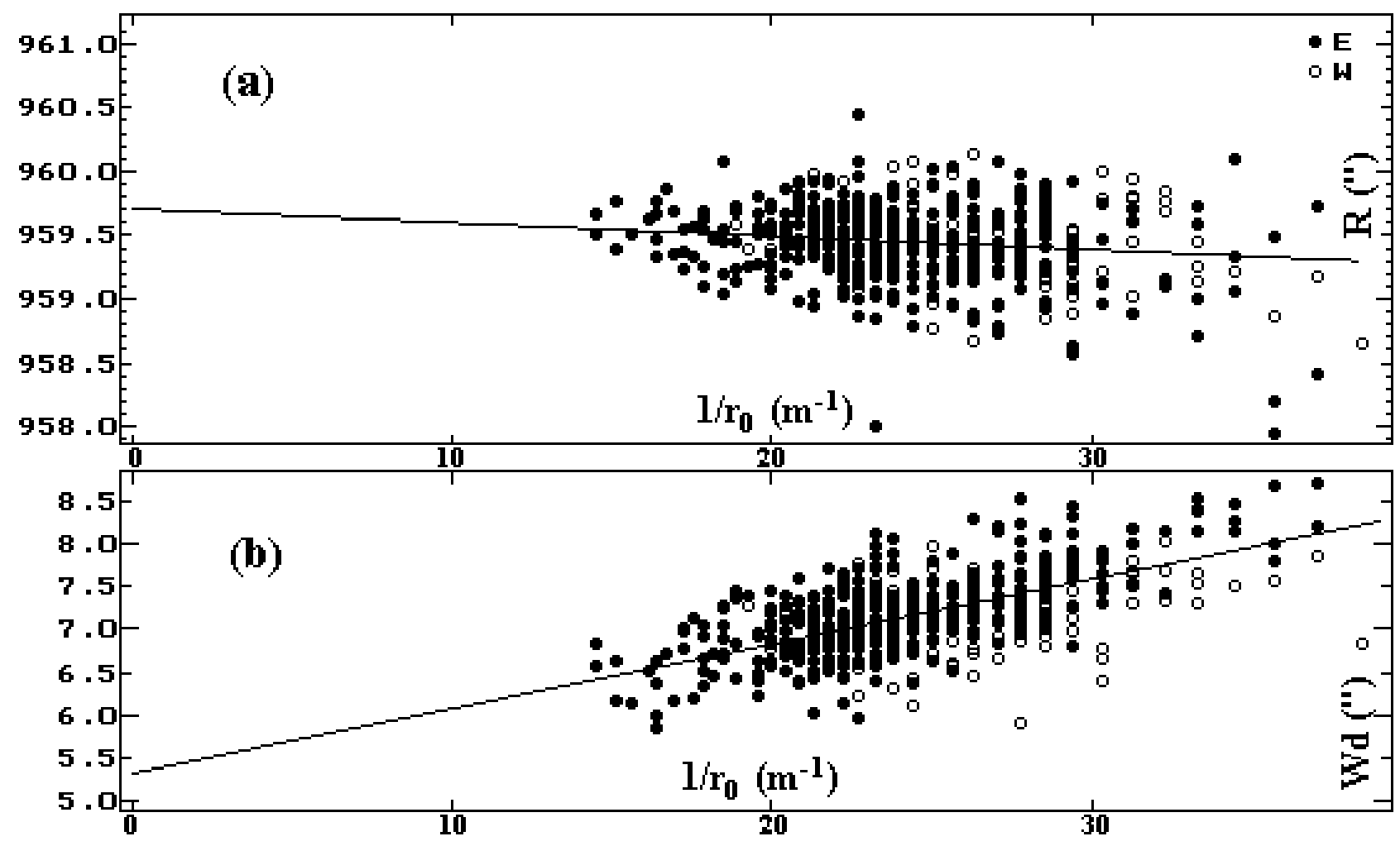

Fig. 1. Derivative methods results: linear fits and extensions for solar radius $(R)$ and derivative width $\left(W_{\mathrm{d}}\right)$ as functions of the Fried parameter $\left(r_{0}\right)$. The value $1 / r_{0}=0 \mathrm{~m}^{-1}$ corresponds to an ideal atmosphere, for which $r_{0} \rightarrow \infty$. a): solar radius and Fried parameter; $\mathbf{b})$ : derivative width and Fried parameter

\section{Determination of the solar radius, following its new definition}

The adopted method gives very good and homogeneous results but cannot solve easily the problem of the solar limb definition, due to the center to limb darkening. It is well known that the maximum of the derivative along a solar image section define the limb of an apparent Sun, which is not the true limb of the Sun. This effect is the result of the combination of the diffraction, the center to limb darkening and the atmospheric turbulence, which have as result to shift systematically the inflection points towards the center of the Sun image. Consequently, the apparent solar radius is always smaller than the true one (Rösch et al. 1996).

Considering the amount of information obtained during each transit, it seems possible to evaluate this effect and to derive the corresponding corrections to be applied to the results.

The ways to suppress the preceding effects seem to be:

- to extrapolate the results of the numerical analysis by a method which needs to be defined, or

- to construct a model of the solar images, which takes account of all the physical effects.
Only the CCD observations done with the rotating shutter will be considered here. After a precise description of the adopted model, an application to solar measurements made at Calern Observatory will conduct to new corrected results.

The results obtained by numerical derivation of the same data are analyzed and extrapolated. These results allow us to compare the two methods.

\subsection{The numerical derivation and its extension}

Before the construction of the model, the problem led by the center to limb darkening and the atmosphere was known and numerical studies were done to suppress it. With the first reduction method, it was a priori impossible to evaluate directly the necessary corrections. Nevertheless, correlations have been established between the observed radius and other parameters as the Fried parameter given by:

$r_{0}=8.2510^{5} \cdot D^{-\frac{1}{5}} \cdot \lambda^{\frac{6}{5}} \cdot\left(\sigma^{2}\right)^{-\frac{3}{5}}$

which may be considered as a representation of the atmospheric turbulence (Irbah et al. 1994). $D$ is the aperture of the astrolabe refractor, $\lambda$ is the wavelength $(\mu \mathrm{m})$ and $\sigma$ 
is the standard deviation of the linear fit on the observed trajectories.

This formula giving $r_{0}$, which depends on $\sigma$, shows that the quality of the sky is correlated with $r_{0}$, the best sky corresponding to $r_{0} \rightarrow \infty$ or to a null value for $\sigma$.

Another interesting parameter is the derivative width, defined at half height of the derivative peak, or, also, as the distance between the two inflection points of the derivative. We use here the daily mean value of each quantity (Table 1).

The first study shows that the Fried parameter and the width of the numerical derivative are strongly correlated (for more details, see Sinceac 1998; we use here these results only to validate the new method). If $r_{0}$ is the Fried parameter and $W_{\mathrm{d}}$ the width of the derivative obtained numerically, we find that (Fig. 1b):

$W_{\mathrm{d}}=0 . \cdot{ }^{\prime \prime} 07245 \cdot\left(\frac{1}{r_{0}}-24 .^{\mathrm{m}^{-1}} 1188\right)+77^{\prime \prime} 137$.

Excepted the conventional mean value of $\frac{1}{r_{0}}, 24.1188 \mathrm{~m}^{-1}$, the numerical coefficients are respectively known with the standard errors $\pm 0 .^{\prime \prime} \cdot \mathrm{m} 00416$ and $\pm 0{ }^{\prime \prime} 022$ for the constant term.

In the same manner, the correlation between $r_{0}$ and the observed solar radius $R$ was computed (Fig. 1a):

$R=-0 .{ }^{\prime \prime}{ }^{\mathrm{m}} 00719 \cdot\left(\frac{1}{r_{0}}-24 .^{\mathrm{m}^{-1}} 1188\right)+959.455$

with the respective errors on the constants: $\pm 0{ }^{\prime \prime} \cdot{ }^{\mathrm{m}} 00307$ and $\pm 00^{\prime \prime} 013$.

The Fried parameter represents the atmosphere effects that we want to remove. As we know that the ideal atmosphere corresponds to an infinite value for $r_{0}$, it is interesting to examine which the values of $W_{\mathrm{d}}$ and $R$ are when the Fried parameter is extrapolated to $+\infty$. For $\frac{1}{r_{0}}=0$, we obtain:

$R=959^{\prime \prime} 628 \pm 0 . \prime 075$.

This is practically the value given one century ago by Auwers 1891! The derivative width becomes equal to:

$W_{\mathrm{d}}=5^{\prime \prime} .39 \pm 0.10$.

These results may represent the values, obtained with the same instrument, of the solar radius and of the derivative width along the CCD lines, through a perfect atmosphere or above the atmosphere. Due to the extrapolation method, one can see that the precision is not so good.

\subsection{The model}

We have shown that the phenomena conducting to an apparent solar image are, mainly:

- a center to limb darkening of the solar intensity, including absorption and turbulence coming from the terrestrial atmosphere;
- the instrumental diffraction effect.

It is well known that the perfect diffraction curve of an uniform extended source through a circular aperture presents a maximum of its derivative exactly on the true position of the limb of the source surface (Chrétien 1959). If the source intensity is not constant, the position of this point changes and generally does not coincide with the true limb.

We will now consider a CCD line section of the apparent image. Without darkening effect, the representative intensity curve is assumed to be a hyperbolic tangent curve as $\tanh (x)+1$ (the value 1 is added to obtain always positive values). The resulting function (of the form $\left.\frac{2 \mathrm{e}^{x}}{\mathrm{e}^{x}+\mathrm{e}^{-x}}\right)$ is multiplied by a first unknown constant $a$, in order to represent the signal amplitude. The abscissa of the inflection point is obtained for $x=0$, and a change of a parameter is used to put it in $x_{0}$. By the same way, the slope of the intensity function is assumed to be different from 1 and depends on a constant $b$. So the $x$ parameter is changed to $b \cdot\left(x-x_{0}\right)$.

Finally, an unknown constant $c$ is added to give account of the added intensity due to noise and sky lighting.

With these assumptions, the intensity model along a CCD line, due to diffraction effect only, can be written as:

$I_{0}(x)=c+2 \cdot a \cdot \frac{\mathrm{e}^{b\left(x-x_{0}\right)}}{\mathrm{e}^{b\left(x-x_{0}\right)}+\mathrm{e}^{-b\left(x-x_{0}\right)}}$.

In Eq. (1), $x_{0}$ is the abscissa of the point of the solar limb which represents the diameter extremity verifying the adopted definition as this function represents a perfect extended source with constant intensity. In order to give account of the center to limb darkening, we have to adopt a mathematical representation. Here a simple model of this effect is adopted, which has exactly the same form as the perfect model, but with a slope parameter $p$, which has always the same sign as $b$ and is much lower. The final mathematical form of the model consists to multiply the part of the perfect model which contains $a$ by the center to limb darkening model.

The result, more realistic, can be written now:

$I(x)=c+4 a \frac{\mathrm{e}^{b\left(x-x_{0}\right)}}{\mathrm{e}^{b\left(x-x_{0}\right)}+\mathrm{e}^{-b\left(x-x_{0}\right)}} \cdot \frac{\mathrm{e}^{p\left(x-x_{0}\right)}}{\mathrm{e}^{p\left(x-x_{0}\right)}+\mathrm{e}^{-p\left(x-x_{0}\right)}}(2)$ One can remark that the perfect model (Eq. 1) and the one for center to limb darkening have the same position for the inflection point $x_{0}$. This fact has no physical significance and if we adopt a different position for the two inflection points, nothing change in the results, except the complexity of the theoretical and practical calculations. As this model, we will see, is convenient, we do not keep this supplementary unknown.

A second remark may be that far from the limb, these functions do not give an exact representation of the image, and that they can be used only in the vicinity of the limb. In fact, the intensity $I(x)$ increases from $c$ to $c+4 a$, which is not the case for the solar light intensity, which increases 


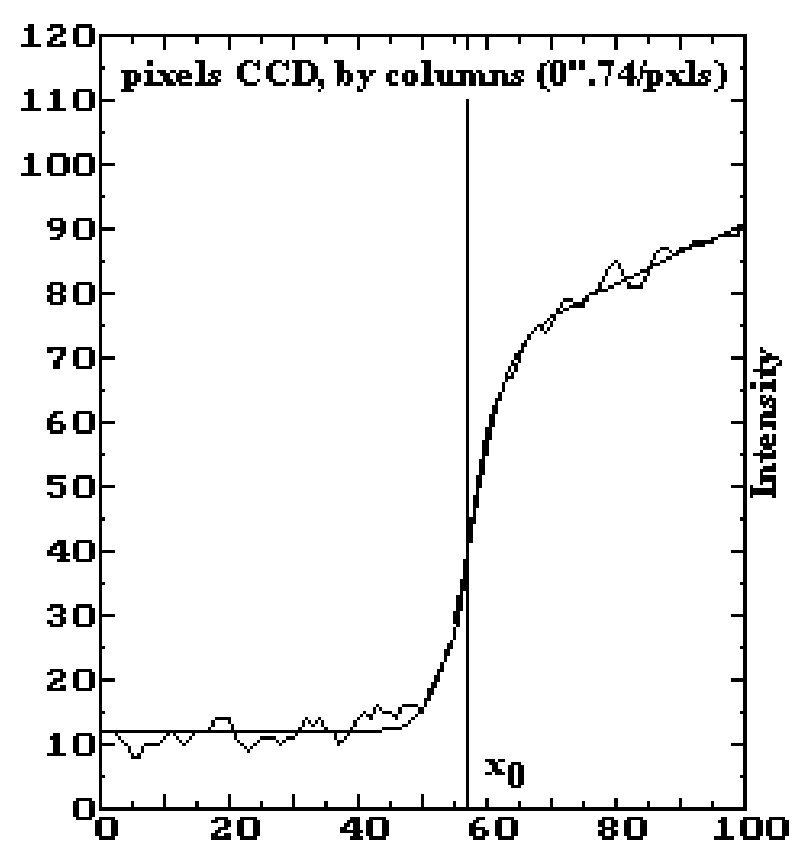

Fig. 2. Section along a CCD line: least squares fitting of the model on real data (the vertical line marks the abscissa of the inflection point, $x_{0}$ ). The vertical scale gives the intensity level of the signal in a scale defined by the CCD camera (intensity levels between 0 and 255)

from a minimum to a maximum and decreases to the minimum. In fact, the entire solar image may be represented by the product of $a$ by two functions of $b, p$ and $x_{0}^{\prime}$ for the first one, and of $-b,-p$ and $x_{0}^{\prime \prime}$ for the second one (Eq. 4). In this case, in the vicinity of, saying $x_{0}^{\prime}$, the functions which contain $x_{0}^{\prime \prime}$ are practically equal to 1 , and vice versa. As, on the CCD, the field is very little and contains only one of the two possible inflection points, we are always in the preceding situation and, consequently, we will use the simple Eqs. (2) or (3), by changing the $b$ and $p$ signs following the observed part of the Sun.

We give, in the Appendix, all the necessary expressions needed to use this model. The notations adopted in Appendix will be used in the following.

Now, it is necessary to know where is the abscissa of the zero of the second derivative of the perfect and realistic model. Starting with the Eq. (1), the zero of the second derivative is given by the equation $\frac{\mathrm{d}^{2} U}{\mathrm{~d} x^{2}}=0$ which is equivalent (see Appendix) to:

$X-\frac{1}{X}=0$

This function is equal to zero if $X^{2}=1$ or $x=x_{0}$ following the expression of $X$. The ideal function, without darkening, reaches its extremum in $x_{0}$.

Now, the final model, given by Eqs. (2) or (3) has to be derived in order to find the abscissa of the inflection point. The second derivative of this function is given by the Eqs. (5) or (6).
The terms containing second derivatives are equal to zero for $x=x_{0}$, but the term $\frac{\mathrm{d} U}{\mathrm{~d} x} \cdot \frac{\mathrm{d} V}{\mathrm{~d} x}$ is different from zero.

We can conclude now that the inflection point abscissa is not the same in the Eqs. (1) and (2). As the interesting parameter is $x_{0}$, one way to get it is to solve the Eq. (6) for which the solution is function of the parameters $x_{0}$, $b$ and $p$ only. This solution $x_{\mathrm{s}}$ will contain the difference with the true value $x_{0}$ and solve the problem, provided that $b$ and $p$ are known. It seems better to fit, by least squares method, the model on the data obtained along a line. This method gives directly $x_{0}$ and the others parameters. Figure 2 shows a section on a CCD line and the model obtained by this method. Anyway, an approximate solution of the Eq. (6) was calculated to evaluate the difference $x_{\mathrm{s}}-x_{0}$, assuming that this difference is little as $b$ and $p$ are small and as, normally, $|p|<\frac{|b|}{10}$. The calculation, made at the first order of the parameters (see Appendix for more precise formula), gives:

$x_{\mathrm{s}}-x_{0}=\frac{p}{b^{2}} \quad$ or $\quad x_{\mathrm{s}}=x_{0}+\frac{p}{b^{2}}$.

In the model, $b$ and $p$ have always the same sign. If $p$ is negative, the center of the Sun is in the direction of the negative $x$ and $x_{\mathrm{s}}<x_{0}$, and the contrary if $p>0$. This shows that, as it was write previously, the apparent diameter of the Sun is always smaller than the true one. With the usual values of $b \simeq 0.15$ and $p \simeq 0.01$ the difference between real and apparent radius is approximately equal to 0.44 pixel or $\simeq 0^{\prime \prime} .33$.

Considering the high value of this effect, it seems much more convenient to determine the model parameters, by fitting it to the data and using the whole useful data along the CCD line (which turns around 80 kept values for the intensities). The main result is that, effectively, the new obtained radius are greater than the previous ones obtained by numerical research of the first derivative extremum.

\section{Results}

In this observational mode, where the two images are separated, each CCD frame contains only the direct or the reflected image of the Sun. The acquired data are contained in a window of 256 lines $\times 101$ columns. A first least squares fitting along each line uses only 101 values of the intensities to determine the parameters $x_{0}, a, b, p$ and $c$ of the model.

The calculation of the observed radius follows the same method used after numerical determination of the inflection points (Sinceac 1998; Sinceac et al. 1998a).

The Table 1 gives all the results obtained by observations done with the rotating shutter. They are given for the first method of reduction (position of the extremum of the first derivative) and for the method using the model. In each image, the fact that the CCD window (only 101 
Table 1. 1996-1997 CCD measurements campaign, daily mean results

\begin{tabular}{|c|c|c|c|c|c|c|c|c|c|c|c|}
\hline \multicolumn{3}{|c|}{ Date } & $\mathbf{N}$ & $\begin{array}{l}\text { Solar Radius } \\
\text { Derivative }\left({ }^{\prime \prime}\right)\end{array}$ & $\begin{array}{c}\varepsilon \\
\left({ }^{\prime \prime}\right)\end{array}$ & $\begin{array}{l}\text { Solar Radius } \\
\text { Model (") }\end{array}$ & $\begin{array}{c}\varepsilon \\
\left({ }^{\prime \prime}\right)\end{array}$ & $\begin{array}{c}\mathbf{b} \\
\left(\text { pixel }^{-1}\right)\end{array}$ & $\begin{array}{c}\varepsilon \\
\left(\text { pixel }^{-1}\right)\end{array}$ & $\begin{array}{c}\mathbf{p} \\
\left(\text { pixel }^{-1}\right)\end{array}$ & $\begin{array}{c}\varepsilon \\
\left(\text { pixel }^{-1}\right)\end{array}$ \\
\hline 16 & Jul. & 1996 & 10 & 959.38 & 0.11 & 959.47 & 0.13 & 0.1423 & 0.0031 & 0.00930 & 0.00040 \\
\hline 17 & Jul. & 1996 & 1 & 959.81 & - & 959.97 & - & 0.1594 & - & 0.01004 & - \\
\hline 23 & Jul. & 1996 & 10 & 959.27 & 0.08 & 959.51 & 0.10 & 0.1485 & 0.0030 & 0.00975 & 0.00016 \\
\hline 24 & Jul. & 1996 & 3 & 959.36 & 0.05 & 959.53 & 0.12 & 0.1594 & 0.0094 & 0.00964 & 0.00033 \\
\hline 26 & Jul. & 1996 & 3 & 959.35 & 0.16 & 959.69 & 0.19 & 0.1468 & 0.0026 & 0.01037 & 0.00023 \\
\hline 29 & Jul. & 1996 & 9 & 959.38 & 0.04 & 959.56 & 0.08 & 0.1494 & 0.0024 & 0.00934 & 0.00019 \\
\hline 31 & Jul. & 1996 & 9 & 959.35 & 0.13 & 959.58 & 0.09 & 0.1448 & 0.0040 & 0.00934 & 0.00020 \\
\hline 01 & Aug. & 1996 & 13 & 959.43 & 0.06 & 959.66 & 0.06 & 0.1543 & 0.0024 & 0.01005 & 0.00010 \\
\hline 22 & May & 1997 & 15 & 959.37 & 0.09 & 959.50 & 0.09 & 0.1463 & 0.0020 & 0.00931 & 0.00013 \\
\hline 23 & May & 1997 & 8 & 959.44 & 0.08 & 959.53 & 0.12 & 0.1465 & 0.0047 & 0.00901 & 0.00016 \\
\hline 24 & May & 1997 & 7 & 959.32 & 0.09 & 959.50 & 0.10 & 0.1491 & 0.0035 & 0.00939 & 0.00017 \\
\hline 26 & May & 1997 & 15 & 959.42 & 0.06 & 959.61 & 0.07 & 0.1464 & 0.0027 & 0.00946 & 0.00017 \\
\hline 27 & May & 1997 & 19 & 959.46 & 0.08 & 959.66 & 0.09 & 0.1481 & 0.0029 & 0.00971 & 0.00018 \\
\hline 28 & May & 1997 & 15 & 959.35 & 0.06 & 959.60 & 0.08 & 0.1411 & 0.0047 & 0.00935 & 0.00020 \\
\hline 29 & May & 1997 & 10 & 959.47 & 0.08 & 959.80 & 0.09 & 0.1292 & 0.0019 & 0.00916 & 0.00009 \\
\hline 30 & May & 1997 & 9 & 959.40 & 0.07 & 959.60 & 0.13 & 0.1467 & 0.0038 & 0.00928 & 0.00012 \\
\hline 03 & Jun. & 1997 & 5 & 959.07 & 0.30 & 959.27 & 0.23 & 0.1354 & 0.0086 & 0.00849 & 0.00046 \\
\hline 07 & Jun. & 1997 & 5 & 958.99 & 0.24 & 959.34 & 0.13 & 0.1384 & 0.0082 & 0.00986 & 0.00013 \\
\hline 15 & Jun. & 1997 & 8 & 959.38 & 0.06 & 959.46 & 0.05 & 0.1603 & 0.0032 & 0.00943 & 0.00016 \\
\hline 21 & Jun. & 1997 & 9 & 959.54 & 0.03 & 959.74 & 0.08 & 0.1518 & 0.0049 & 0.00940 & 0.00021 \\
\hline 23 & Jun. & 1997 & 19 & 959.57 & 0.04 & 959.71 & 0.05 & 0.1435 & 0.0019 & 0.00921 & 0.00008 \\
\hline 24 & Jun. & 1997 & 8 & 959.49 & 0.09 & 959.62 & 0.09 & 0.1445 & 0.0033 & 0.00910 & 0.00017 \\
\hline 25 & Jun. & 1997 & 10 & 959.44 & 0.11 & 959.54 & 0.11 & 0.1542 & 0.0023 & 0.00952 & 0.00017 \\
\hline 28 & Jun. & 1997 & 9 & 959.34 & 0.10 & 959.45 & 0.11 & 0.1451 & 0.0030 & 0.00913 & 0.00017 \\
\hline 13 & Aug. & 1997 & 5 & 959.34 & 0.08 & 959.56 & 0.06 & 0.1580 & 0.0034 & 0.01034 & 0.00021 \\
\hline 14 & Aug. & 1997 & 9 & 959.63 & 0.06 & 959.81 & 0.05 & 0.1602 & 0.0026 & 0.00996 & 0.00022 \\
\hline 15 & Aug. & 1997 & 8 & 959.47 & 0.06 & 959.71 & 0.09 & 0.1599 & 0.0031 & 0.01017 & 0.00012 \\
\hline 18 & Aug. & 1997 & 8 & 959.34 & 0.11 & 959.56 & 0.08 & 0.1462 & 0.0043 & 0.00943 & 0.00023 \\
\hline 19 & Aug. & 1997 & 7 & 959.51 & 0.06 & 959.74 & 0.05 & 0.1565 & 0.0020 & 0.01005 & 0.00017 \\
\hline 22 & Aug. & 1997 & 3 & 959.41 & 0.24 & 959.93 & 0.15 & 0.1233 & 0.0049 & 0.00902 & 0.00030 \\
\hline 23 & Aug. & 1997 & 7 & 959.47 & 0.08 & 959.68 & 0.07 & 0.1506 & 0.0039 & 0.00971 & 0.00024 \\
\hline 24 & Aug. & 1997 & 8 & 959.64 & 0.09 & 959.86 & 0.08 & 0.1559 & 0.0037 & 0.00985 & 0.00022 \\
\hline 25 & Aug. & 1997 & 3 & 959.47 & 0.08 & 959.70 & 0.08 & 0.1546 & 0.0093 & 0.00995 & 0.00046 \\
\hline 27 & Aug. & 1997 & 5 & 959.52 & 0.16 & 959.71 & 0.16 & 0.1447 & 0.0051 & 0.00941 & 0.00011 \\
\hline 29 & Aug. & 1997 & 11 & 959.70 & 0.09 & 959.94 & 0.10 & 0.1436 & 0.0020 & 0.00981 & 0.00012 \\
\hline 30 & Aug. & 1997 & 14 & 959.33 & 0.09 & 959.61 & 0.11 & 0.1373 & 0.0030 & 0.00937 & 0.00013 \\
\hline 31 & Aug. & 1997 & 14 & 959.47 & 0.05 & 959.62 & 0.04 & 0.1523 & 0.0036 & 0.00972 & 0.00013 \\
\hline 01 & Sep. & 1997 & 10 & 959.44 & 0.07 & 959.70 & 0.07 & 0.1502 & 0.0055 & 0.01004 & 0.00023 \\
\hline 04 & Sep. & 1997 & 6 & 959.47 & 0.04 & 959.61 & 0.06 & 0.1592 & 0.0046 & 0.00983 & 0.00016 \\
\hline 05 & Sep. & 1997 & 7 & 959.57 & 0.12 & 959.72 & 0.13 & 0.1598 & 0.0039 & 0.01033 & 0.00021 \\
\hline 06 & Sep. & 1997 & 7 & 959.44 & 0.05 & 959.63 & 0.09 & 0.1641 & 0.0056 & 0.01031 & 0.00050 \\
\hline 07 & Sep. & 1997 & 6 & 959.54 & 0.19 & 959.73 & 0.22 & 0.1526 & 0.0080 & 0.00944 & 0.00036 \\
\hline 08 & Sep. & 1997 & 10 & 959.42 & 0.07 & 959.59 & 0.06 & 0.1592 & 0.0037 & 0.01005 & 0.00011 \\
\hline 09 & Sep. & 1997 & 14 & 959.59 & 0.05 & 959.73 & 0.08 & 0.1569 & 0.0032 & 0.00985 & 0.00017 \\
\hline 10 & Sep. & 1997 & 11 & 959.52 & 0.09 & 959.68 & 0.08 & 0.1565 & 0.0053 & 0.00982 & 0.00007 \\
\hline 12 & Sep. & 1997 & 5 & 959.54 & 0.13 & 959.70 & 0.16 & 0.1580 & 0.0048 & 0.00982 & 0.00018 \\
\hline
\end{tabular}

columns) is not centered on the solar image doesn't affect the results for the derivative method, which uses practically all the CCD lines (the intensity function is derived only in the vicinity of the inflection point, Sinceac 1998). To find the parameters of the model by the second method, the software needs more informations that the first one and more CCD lines will be eliminated, but the model detects better the problems. To be able to compare the two set of results, the two analysis modes use approximately the same data. In fact, the elimination procedure of bad CCD images being not the same, the set of data used for each method cannot be strictly identical (there is a very great quantity of data). Finally, it is the method of least squares used for the parabolas on each image 
which decides possible removal of lines and/or images (Sinceac 1998). This explain why the results by derivative method given here are not exactly the same as the ones published in our precedent paper (Sinceac et al. 1998a) and on the Table 1. The mean values are:

$$
\begin{aligned}
& R=959 . .44 \pm 0 . \text { '02 } \quad \text { by derivative method alone; } \\
& R=959 . .64 \pm 0 . \text { with the model. }
\end{aligned}
$$

The dispersion $\sigma$ of the results is practically the same with the two methods and equal to $0^{\prime \prime} 14$.

Using the extrapolation for $r_{0} \rightarrow \infty$ in the derivative method, we have:

$$
R=959^{\prime \prime} 63 \pm 0 . \text {. } 08
$$

which is not so precise.

The difference found between the radius obtained by each method shows that the values of the constants $b$ and $p$ are not far from the ones given in the preceding paragraph. In fact, during the calculations, the values encountered for $b$ and $p$ turn respectively around 0.15 and 0.01 pixel $^{-1}$. But few variations appear, due mainly to the atmosphere and the integration time of each image $(0.02$ second).

It is interesting to compare these values with the theoretical results obtained using the model. We have just seen that the mean solar radius, obtained with the derivative method and extrapolated for $r_{0} \rightarrow \infty$, becomes practically the same as the one obtained with the model. The successive correlations calculated between the correction to the apparent inflection point $x_{\mathrm{s}}$ (Appendix) and the new computed $r_{0}$ give, in the same units:

$x_{\mathrm{s}}-x_{0}=\frac{p}{b^{2}}=0.01394 \cdot\left(\frac{1}{r_{0}}-23.4369\right)+0.4322$

with the respective errors \pm 0.00055 and \pm 0.0032 .

The new derivative width is also correlated with $r_{0}$ and we have:

$W_{\mathrm{m}}=0.1798 \cdot\left(\frac{1}{r_{0}}-23.4369\right)+9.272$

with the respective errors: \pm 0.0066 and \pm 0.039 . The calculation for $\frac{1}{r_{0}}=0$ gives:

$\frac{p}{b^{2}}=0.105 \pm 0.013 \quad$ and $\quad W_{\mathrm{m}}=5^{\prime \prime} .06 \pm 0.04$.

The successive model derivatives show that the theoretical derivative width $w_{\mathrm{h}}$ is equal to $\frac{1.8708}{b}$, in pixel (Appendix). The preceding result being identified with $w_{\mathrm{h}}$, it becomes possible to calculate $b$ from the theoretical value of $w_{\mathrm{h}}$. As the pixel value is $0^{\prime \prime} .74$, we obtain (in pixel $\left.^{-1}\right)$ :

$b=0.2736 \pm 0.0028$.

Immediately, the value of $\frac{p}{b^{2}}$ give us the one of $p$ (in pixel $\left.^{-1}\right)$ :

$p=0.0079 \pm 0.0016$.
The obtained value for $b$ is much greater than the mean value calculated with the data of the Table 1 . If, by the model, we research the maximum value of the slope of the curve representing the intensities along a CCD line, we obtain $a \cdot(b+p)$. The obtained high value for $b$ may be interpreted as for a perfect or outside the atmosphere, where, logically, this parameter is much greater than the one detected through the real atmosphere.

For the parameter $p$, the result is opposite: the value outside the atmosphere is less than the observed one. This result is also logical as the center to limb darkening effect can be essentially increased by the transmission of the terrestrial (and solar?) atmosphere and not so much by its turbulence.

Considering the relatively little amount of data, the information obtained here are sufficiently precise to find coherent results, and give very interesting first information about the disruptive effects introduced by the atmosphere. The Table 1 shows also that the parameters $b$ and $p$ are finally relatively stable since the $b$ values stand between 0.129 and 0.164 and the $p$ values between 0.0090 and 0.0103 .

We can consider now that $b=0.274 \pm 0.003$ is, outside the atmosphere, the value of the main parameter defining the solar light intensity slope near the inflection point of the limb. In the same conditions, the parameter $p=0.0079 \pm 0.0016$ represents the slope parameter of the center to limb effect, near the limb. Finally, the solar radius, corrected for the atmospheric and darkening effects is equal to:

$9599^{\prime \prime} 64 \pm 0.0^{\prime \prime} 02$ using the model;

$9599^{\prime \prime} 63 \pm 0$.' 08 by extrapolation to $r_{0} \rightarrow \infty$

in the numerical derivation.

\section{Comparison with others results}

Several authors published mean values for the apparent solar radius, measured with different instruments and methods. Among those there, we kept solely here results of the most recent measure sets, and that are comparable to ours (Table 2) ${ }^{1}$.

Concerning the solar astrolabe of Calern, a recent analysis presents visual and CCD measures results done in parallel on the same instrument, between 1996 and 1998 (Laclare et al. 1999). For the visual measures, the observed solar limb would be in shrinking in relation to the real limb, in proportion to the zenith distance of the observation. This effect, due to the fact that the eye appreciates the solar side for a threshold of light intensity, that varies with the atmospheric absorption (therefore, largely, with zenithal distance of observation), duct to an obvious

\footnotetext{
1 The sources for the results obtained at Rio de Janeiro and Calern Observatories (visual and CCD observations) are http://on.br/radius.html and http://obs-nice.fr/radius
} 
Table 2. Some recent results for the apparent solar radius, obtained by different methods and instruments (annual and/or general mean values)

\begin{tabular}{|c|c|c|c|c|c|}
\hline Instrument / Method & Remarks & $\mathbf{N}$ & Period & Result & Author / Reference \\
\hline $\begin{array}{l}\text { Drift-Scan CCD } \\
\text { Drift-Scan Visual }\end{array}$ & & $\begin{array}{l}126 \\
427\end{array}$ & $\begin{array}{l}1996 \\
1996\end{array}$ & $\begin{array}{l}959^{\prime \prime} 73 \pm 0^{\prime \prime} 05 \\
960^{\prime \prime} 53 \pm 0 . .02\end{array}$ & Wittmann 1997 \\
\hline $\begin{array}{l}\text { Solar Disk Sextant } \\
\text { Fast Fourrier Transform } \\
\text { Definition (FFTD) }\end{array}$ & & $\begin{array}{l}1 \mathrm{fly} \\
1 \mathrm{fly}\end{array}$ & $\begin{array}{l}1990 \\
1992\end{array}$ & $\begin{array}{l}959^{\prime \prime} 60 \pm 0.0^{\prime \prime} 17 \\
959^{\prime \prime} 53 \pm 0 . .09\end{array}$ & $\begin{array}{l}\text { Maier et al. } 1992 \text {, } \\
\text { Sofia et al. } 1994\end{array}$ \\
\hline Limb-Darkening Scans & & 72 & 1981-90 & $959^{\prime \prime} 62 \pm 0.0^{\prime \prime} 03$ & Neckel 1995 \\
\hline $\begin{array}{l}\text { Solar Diameter Monitor } \\
\text { (photoelectrical) FFTD }\end{array}$ & & & $1981-87$ & $959^{\prime \prime} 68 \pm 0.0^{\prime \prime} 02$ & Brown \& Christensen-Dalsgaard 1998 \\
\hline $\begin{array}{l}\text { Solar CCD Astrolabe } \\
\text { full pupil, without shutter } \\
\text { San Fernando Observatory }\end{array}$ & optical square & 100 & 1998 & $959^{\prime \prime} 33 \pm 0^{\prime \prime} 04$ & Sánchez 1998 \\
\hline $\begin{array}{l}\text { Solar Visual Astrolabe } \\
\text { Malatya Observatory }\end{array}$ & 2 prisms & 170 & 1993-96 & $959^{\prime \prime} 44 \pm 0.0^{\prime \prime} 05$ & Kiliç 1998 \\
\hline $\begin{array}{l}\text { Solar Visual Astrolabe } \\
\text { Santiago Observatory }\end{array}$ & 2 prisms & $\begin{array}{l}123 \\
120 \\
822\end{array}$ & $\begin{array}{c}1996 \\
1997 \\
1990-97\end{array}$ & $\begin{array}{l}959^{\prime \prime} 85 \pm 0^{\prime \prime} 03 \\
960^{\prime \prime} 00 \pm 0^{\prime \prime} 03 \\
960^{\prime \prime} 39 \pm 0^{\prime \prime} 13\end{array}$ & Noël 1998 \\
\hline $\begin{array}{l}\text { Solar CCD Astrolabe } \\
\text { Variable Angle Prism } \\
\text { without rotating shutter } \\
\text { Rio de Janeiro Observatory }\end{array}$ & $\begin{array}{l}\text { East } \\
\text { West }\end{array}$ & $\begin{array}{l}3500 \\
2600\end{array}$ & $\begin{array}{l}1996-97 \\
1996-97\end{array}$ & $\begin{array}{l}959^{\prime \prime} 20 \pm 0^{\prime \prime} 02 \\
959 . .14 \pm 0^{\prime \prime} .03\end{array}$ & $\begin{array}{l}\text { Jilinski et al. } 1998, \\
\text { Jilinski et al. } 1999\end{array}$ \\
\hline $\begin{array}{l}\text { Calern Solar Astrolabe } \\
\text { CCD Derivative } \\
\text { CCD Wavelet + Derivative }\end{array}$ & & $\begin{array}{l}348 \\
348\end{array}$ & $\begin{array}{l}1996-97 \\
1996-97\end{array}$ & $\begin{array}{l}959^{\prime \prime} .45 \pm 00^{\prime \prime} 01 \\
959^{\prime \prime} 53 \pm 00^{\prime \prime} 01\end{array}$ & $\begin{array}{l}\text { Sinceac } 1998 \\
\text { Irbah } 1998\end{array}$ \\
\hline $\begin{array}{l}\text { CCD Model } \\
\text { CCD Derivative }\end{array}$ & $\frac{1}{r_{0}} \rightarrow 0$ & $\begin{array}{l}409 \\
409\end{array}$ & $\begin{array}{l}1996-97 \\
1996-97\end{array}$ & $\begin{array}{l}959^{\prime \prime} 64 \pm 00^{\prime \prime} 02 \\
959^{\prime \prime} 63 \pm 00^{\prime \prime} 08\end{array}$ & $\begin{array}{l}\text { This article } \\
\text { Sinceac } 1998\end{array}$ \\
\hline $\begin{array}{l}\text { CCD Derivative } \\
\text { Visual }\end{array}$ & $\begin{aligned} \sigma_{t} & \rightarrow 0 \\
z & \rightarrow 0\end{aligned}$ & $\begin{array}{l}592 \\
418\end{array}$ & $\begin{array}{l}1996-98 \\
1996-98\end{array}$ & $\begin{array}{l}959^{\prime \prime} .59 \pm 0^{\prime \prime} 01 \\
959^{\prime \prime} 60 \pm 0^{\prime \prime} .01\end{array}$ & $\begin{array}{l}\text { Sinceac } 1998 \\
\text { Laclare et al. } 1999\end{array}$ \\
\hline
\end{tabular}

smaller solar radius that the real one. While extrapolating the raw visual measures to the zenith, the mean value of the apparent solar radius becomes identical to the one gotten by the CCD measures extrapolate to a null standard deviation of the points on the trajectories. The last two values presented on Table 2 take account of these corrections (Laclare et al. 1999).

In another work (Bouzaria \& Moussaoui 1996; Irbah 1998) the CCD images have been cleaned by a wavelet analysis before the numeric derivation. This test shows that an adequate cleaning of images improves of meaningful manner the definition of the position of the solar limb (still by numeric derivation), but doesn't give any supplementary information to value other corrections. These information are now gotten thanks to the use of the model, that conducts directly to the solar radius freed of disruptive effects mentioned in the previous chapters. As all these effects have as result a decrease of the solar radius, the use of the model always gives a result closer to the real solar radius, contrary to other methods. We project to apply our model method to these cleaned images.

The measures made with the other solar astrolabe analyzed here are coherent, to the unique exception of measures of Santiago, which presents a relatively large shift comparatively to the others results. The results of the measures made with the astrolabe of Rio de Janeiro (observations without rotating shutter) are affected by an East - West effect, whose possible reasons would be the better quality of the atmosphere for observations to the East, or a bad functioning of the variable angle prism. Besides, at Calern, tests made by the authors with the variable angle prism put in evidence the same effect (Sinceac 1998). The solar astrolabe of San Fernando is a full pupil astrolabe, using one optical square instead prisms, and makes CCD measures without rotating 
shutter. Finally, the polyvalent astrolabe of Malatya will change the site and will function in CCD mode in 1999, with the same software than the Calern one.

Let's notice that the apparent solar radius variation is identical for all measures done with astrolabes (except Santiago), and in anticorrelation with the magnetic cycle of the Sun (Laclare et al. 1996; Jilinski et al. 1999). The differences obtained between the contemporary values could be owed to differences of quality of the atmosphere according to sites. Thus, the scale of mean result sizes obtained seems to indicate the order of the quality of astrolabe sites: the CCD images obtained to Calern show that the derivative width to half-height $\left(W_{\mathrm{h}}\right)$ is smallest, therefore a better atmosphere quality and an obvious bigger solar radius. The utilization of the model for the reduction of measures done with every solar astrolabe could improve the consistency of results.

The results of measures of Drift-Scan $(\lambda=475.8 \mathrm{~nm})$, are row outputs, no corrected of atmospheric turbulence effects (Wittmann 1997). The big gap between the visual and CCD measures could be due to differences between the respective definitions of the solar limb. Besides, the big focal length of this instrument could generate errors which are difficult to evaluate.

Finally, measures done with Solar Disk Sextant, Solar Diameter Monitor and those of Limb-Darkening are freed of the atmospheric effects, by the reduction method used (Finite Fourrier Transform Definition), and one can notice that obtained results are nearly identical to those obtained by the model.

\section{Conclusion}

We have seen that the use of the model (which can be considered as an interpolating function) gives results in agreement with many other observations studied here, which are also corrected for the disruptive effects. In the same manner, the results obtained by the derivative method not extrapolated agree with the others results not corrected, or with the visual observations.

The fundamental difference between the two methods is a different use of the data. The relevant parameter, which is the abscissa of the derivative extremum, is obtained in two different ways:

1. By numerical method, an approximate detection is done, followed by a precise numerical computation around the approximate position. Only few data are used in this final computation, around 40 pixels around the first approximate value. On this interval, the abscissa of the derivative extremum is computed by a barycentric method, in which the points where the intensity is greater than half the height of the derivative peak are taken into account. The final result is obtained using the Fried parameter, determined by statistical method, in correlation with others parameters as the width of the derivative and the solar radius. $A$ following extrapolation to the limit value of $r_{0}$ gives the final value of the radius;

2. By the model, a maximum of intensity values along a CCD line are taken into account in order to find the model parameters. But, when the approximate inflection point is too closed to the extremities of the line, the least squares fit program cannot run correctly and the corresponding line is eliminated. Nevertheless, the use of the model gives directly the good value of the solar radius. One can see that the computation of $r_{0}$ and of the derivative width is not used to obtain the correct radius but only to evaluate the perturbing effects.

A new remark which can be done, is that, despite the relatively low precision obtained for the true radius determination by the derivative method (mainly due to the extrapolation), it is very satisfactory to obtain practically the same result with the two methods which are, in theirs principles, completely different.

Another remark turns around the extrapolation done by numerical computation. These results agree very well with the model results, despite the hypothesis we have to assume, which are:

- the parameter $r_{0}$ represents correctly the disruptive effect of the atmosphere;

- the extrapolation, founded on its infinite value for a perfect atmosphere, is valid.

It was not clear that the $r_{0}$ parameter, which is obtained by statistical information about the solar trajectory, could permit to extrapolate the results to $r_{0} \rightarrow \infty$ and conduct to the same results as with the model. Certainly, the amount and the quality of data obtained during several short campaigns are strong favorable factors. Nevertheless, the use of the model do not need to use statistical data. The correct results are immediately obtained with less error $\left(0{ }^{\prime \prime} 02\right.$ in place of $\left.0{ }^{\prime \prime} 08\right)$. The second difference is that the model uses, along each CCD line, the maximum possible of data to calculate the five parameters $a, b, p, x_{0}$ and $c$. The parameters $a$ and $c$ cannot be use here as they contain photometric information about the solar intensity and that photometric reference is not observed.

In our opinion, we think that it is better to use the model for several reasons. Firstly, there is a better use of the data along the CCD line, that implies that the determination of the inflection points should be better. Effectively, the least squares fit precision in the parabola fitting - made to determine the reference point, extremity of the vertical radius - is better when the model is used. Secondly, the Fried parameter, determined with $\sigma$ obtained for the reference point trajectories is less affected by the preceding successive calculations, and particularly the one done to find the derivative peak abscissa. 
Finally, the quality of the CCD observations allows us to have an evaluation of the disruptive effects existing in the observations and to obtain the correct value of the solar radius, which obeys to the assumed definition. The two reduction methods used give the same result, provided that the numerical calculation of the derivative can be correctly extrapolated. But, due to this extrapolation, the result obtained by this method is more precise. We have also, perhaps, the possibility to correct the old observations. It is what we wish.

In a future work we hope to give new results obtained by observations done at Calern Observatory without rotating shutter. The images are much better that the ones obtained with the shutter and analyzed here. So, we hope to have also better results, and to be able to analyze carefully the observations made in Rio de Janeiro and San Fernando Observatories, with which we have very good cooperation, and the future ones made at Antalya (Turkey) where a new campaign will begin in 1999 with a CCD astrolabe.

\section{Appendix}

In the following the notations $X=\mathrm{e}^{b \cdot\left(x-x_{0}\right)}$ and $Y=$ $\mathrm{e}^{p \cdot\left(x-x_{0}\right)}$ will be used, as well as the functions $U=\frac{X}{X+\frac{1}{X}}$ and $V=\frac{Y}{Y+\frac{1}{Y}}$. In these notations the intensity $I(x)$ in Eq. (2) can be written as:

$I(x)=c+4 \cdot a \cdot U \cdot V$.

It is necessary, now, to specify that this model cannot fit the entire section of the real solar image by each CCD line as this function stretch to $c+4 \cdot a$ or to $c$ when $x$ stretch to $\pm \infty$, following the sign of $b$.

To have a correct representation of a CCD line section on the image, we can use the better representation:

$I(x)=c+16 \cdot a \cdot U_{0} \cdot V_{0} \cdot U_{1} \cdot V_{1}$

where the function $4 \cdot U_{1} \cdot V_{1}$ contains the same parameters as $U_{0}$ and $V_{0}$ but with opposite signs for $b$ and $p$ and a different value for the $x_{0}$, as $x_{0}^{\prime}$. The difference $\left|x_{0}^{\prime}-x_{0}\right|$ is nothing else that the solar radius when the section is obtained along a line which contains the solar center. This model (Eq. 4) gives a very satisfactory representation of the solar image sections but is unnecessary in our case because the images obtained by CCD observations are always near the limb of the Sun (the total field of the CCD covers about $6^{\prime}$ ). When the CCD is entirely cover by the Sun image one can see only about $20 \%$ of the total solar image. In this case the function $4 \cdot U_{1} \cdot V_{1}$ (or $4 \cdot U \cdot V$ following the observed part of the limb) is practically constant and equal to 1 , and the differences between the models 3 and 4 around the inflection point is, with a large approximation, equal to 0 . Consequently, we will use the model given by Eqs. (2) or (3) in the following.
The necessary formula to find the limb position, as defined, are the successive derivatives of the Eq. (3). They are:

$\frac{\mathrm{d} I}{\mathrm{~d} x}=4 a\left(\frac{\mathrm{d} U}{\mathrm{~d} x} \cdot V+U \cdot \frac{\mathrm{d} V}{\mathrm{~d} x}\right)$

or, as $\frac{\mathrm{d} X}{\mathrm{~d} x}=b \cdot X$, and the same for $\frac{\mathrm{d} Y}{\mathrm{~d} x}$, with $p$, becomes:

$\frac{\mathrm{d} I}{\mathrm{~d} x}=8 a\left(\frac{b}{\left(X+\frac{1}{X}\right)^{2}} \cdot \frac{Y}{Y+\frac{1}{Y}}+\frac{p}{\left(Y+\frac{1}{Y}\right)^{2}} \cdot \frac{X}{X+\frac{1}{X}}\right)$.

The second derivative can be written as:

$\frac{\mathrm{d}^{2} I}{\mathrm{~d} x^{2}}=4 a\left(V \cdot \frac{\mathrm{d}^{2} U}{\mathrm{~d} x^{2}}+2 \cdot \frac{\mathrm{d} U}{\mathrm{~d} x} \cdot \frac{\mathrm{d} V}{\mathrm{~d} x}+U \cdot \frac{\mathrm{d}^{2} V}{\mathrm{~d} x^{2}}\right)$

or, after calculation:

$$
\begin{aligned}
\frac{\mathrm{d}^{2} I}{\mathrm{~d} x^{2}}= & -16 a \cdot\left(b^{2} \cdot \frac{X-\frac{1}{X}}{\left(X+\frac{1}{X}\right)^{3}} \cdot \frac{Y}{Y+\frac{1}{Y}}\right. \\
& -2 b p \cdot \frac{1}{\left(X+\frac{1}{X}\right)^{2} \cdot\left(Y+\frac{1}{Y}\right)^{2}} \\
& \left.+p^{2} \cdot \frac{Y-\frac{1}{Y}}{\left(Y+\frac{1}{Y}\right)^{3}} \cdot \frac{X}{X+\frac{1}{X}}\right) .
\end{aligned}
$$

Some quantities as the maximum (or minimum) derivative value or the derivative width need to be calculated. It is quite evident that solving the equation $\frac{\mathrm{d}^{2} I}{\mathrm{~d} x^{2}}=0$ is not easy. One way to obtain approximate values of useful quantities is to expand the series which can represent the model $I(x)$.

The calculation, to the fifth order, gives:

$$
\begin{aligned}
y= & c+a \cdot\left[1+(b+p) \cdot\left(x-x_{0}\right)+b p \cdot\left(x-x_{0}\right)^{2}\right. \\
& -\frac{b^{3}+p^{3}}{3} \cdot\left(x-x_{0}\right)^{3}-\frac{b p \cdot\left(b^{2}+p^{2}\right)}{3} \cdot\left(x-x_{0}\right)^{4} \\
& \left.+\frac{2}{15} \cdot\left(b^{5}+p^{5}\right) \cdot\left(x-x_{0}\right)^{5}+\cdots\right] .
\end{aligned}
$$

If $x_{0}, b, p$ and the other parameters are obtained, it is possible to derive information about the images. By derivation of the preceding formula, which is easy now, until the third order, the derivative extremum may be found by solving iteratively the equation $\frac{\mathrm{d}^{2} I}{\mathrm{~d} x^{2}}=0$. We find that the derivative peak abscissa is:

$x_{\mathrm{s}}=x_{0}+\frac{p}{b^{2}}-\frac{2}{3} \cdot \frac{p^{3}}{b^{4}}$.

In the same way, using the third derivative, the first derivative width can be calculated. This parameter may be defined in two way:

1. The width is defined by the distance between the two points in which the intensity is half of the one of the extremum. We will note it as $w_{\mathrm{h}}$; 
2. The width may be also defined as the distance between the two inflection points of the first derivative. This definition needs to calculate the zeros of the third derivative. We will note it as $w_{\mathrm{p}}$.

We obtain: $w_{\mathrm{h}}=\frac{1}{b} \cdot \sqrt{\frac{7}{2}} \approx \frac{1.871}{b}$ and $w_{\mathrm{p}}=\frac{2}{b}$, exactly.

If we use the perfect model, which represent a perfect solar image, the parameter $p$ is equal to zero (Eq. 1). The same quantities may be computed and, we have naturally $x_{\mathrm{s}}=x_{0}$, so:

$w_{\mathrm{h}}=\frac{1}{b} \ln \left(\frac{\sqrt{2}+1}{\sqrt{2}-1}\right) \quad$ and $\quad w_{\mathrm{p}}=\frac{1}{2 b} \ln \left(\frac{2+\sqrt{3}}{2-\sqrt{3}}\right)$.

which gives $w_{\mathrm{h}}=\frac{1.763}{b}$ and $w_{\mathrm{p}}=\frac{1.317}{b}$.

\section{References}

Allen C.W., 1973, in: Astrophysical Quantities. The Athlone Press, London

Auwers A., 1891, Astron. Nachrichten 3068-128, 361

Bouzaria M., Moussaoui R., 1996, Master Thesis, Université des Sciences et de la Technologie d'Alger

Brown T.M., Christensen-Dalsgaard J., 1998, ApJ 500, L195

Chollet F., 1981, Thèse de Doctorat d'État, Université Pierre et Marie Curie, Paris

Chrétien H., 1959, in: Calcul des combinaisons optiques. Sennac, Paris
Irbah A., 1998 (private communication)

Irbah A., Laclare F., Borgnino J., Merlin G., 1994, Solar Phys. 149, 213

Jilinski E.G., Puliaev S.P., Penna J.L., Andrei A.H., Sinceac V., Chollet F., Delmas C., 1998, A\&AS 130, 317

Jilinski E.G., Puliaev S., Penna J.L., Andrei A.H., Laclare F., 1999, A\&AS 135, 227

Kiliç H., 1998, Ph.D. Thesis, Akdeniz University, Antalya

Kurzynska K, 1997 (private communication)

Laclare F., Demarcq J., Chollet F., 1980, C. R. Acad. Sci. Paris Ser. B 291, 189

Laclare F., Delmas C., Coin J.P., Irbah A., 1996, Solar Phys. 166,211

Laclare F., Delmas C., Sinceac V., Chollet F, 1999, C. R. Acad. Sci. Paris Ser. B, 327, 645

Maier E., Twigg L., Sofia S., 1992, ApJ 389, 447

Neckel H, 1995, Solar Phys. 156, 7

Noël F., 1998, A\&AS 132, 195

Pecker J.C., Schatzman E., 1959, in: Astrophysique Générale. Masson et Cie, Paris

Popescu R., 1998 (private communication)

Rösch J., Rozelot J.P., Deslandes H., Desnoux V., 1996, Solar Phys. 165, 1

Sánchez M., 1998 (private communication)

Sinceac V., 1998, Thèse de Doctorat, Observatoire de Paris

Sinceac V., Chollet F., Laclare F., Delmas C., 1998a, A\&AS 128,605

Sofia S., Heaps W., Twigg L.W., 1994, ApJ 427, 1048

Wittmann A.D., 1997, Solar Phys. 171, 231 